\title{
Fixed-Combination Halobetasol Propionate and Tazarotene in the Treatment of Psoriasis: Narrative Review of Mechanisms of Action and Therapeutic Benefits
}

\author{
Mark G. Lebwohl (D) - Emil A. Tanghetti (D) - Linda Stein Gold (D) • \\ James Q. Del Rosso (D) · Nelly K. Gilyadov · Abby Jacobson
}

Received: April 7, 2021 / Published online: June 9, 2021

(C) The Author(s) 2021

\begin{abstract}
Psoriasis is a lifelong disease associated with cycles of remission and relapse. Topical treatments are the front line of psoriasis therapy for most patients and have antiproliferative, antiinflammatory, and immunosuppressive mechanisms of action. Novel fixed-dose combinations of topical therapeutic agents are becoming increasingly available, leveraging multiple mechanisms of action to improve safety and efficacy with formulations that are easier to use and may allow for the use of lower doses of active ingredients. A fixed-combination lotion containing the potent-to-superpotent corticosteroid halobetasol propionate (HP) and the
\end{abstract}

M. G. Lebwohl ( $\square)$

Icahn School of Medicine at Mount Sinai, 5 East 98th Street, 5th Floor, New York, NY 10029, USA e-mail: Lebwohl@aol.com

\section{E. A. Tanghetti}

Center for Dermatology and Laser Surgery,

Sacramento, CA, USA

L. Stein Gold

Henry Ford Hospital, Detroit, MI, USA

J. Q. Del Rosso

JDR Dermatology Research/Thomas Dermatology, Las Vegas, NV, USA

N. K. Gilyadov · A. Jacobson

Ortho Dermatologics, Bausch Health US, LLC (an Affiliate of Bausch Health Companies Inc.),

Bridgewater, NJ, USA retinoid tazarotene (HP 0.01\%/TAZ 0.045\%) was recently developed using polymeric emulsion technology. This new formulation technology allows for more uniform and efficient delivery of the active ingredients at lower doses than conventional monotherapy formulations of either ingredient while providing enhanced hydration and moisturization. This review provides an up-to-date overview of the therapeutic mechanisms of action of HP and TAZ, the rationale behind the development of HP $0.01 \%$ / TAZ $0.045 \%$ lotion, and clinical trials data on the efficacy, safety and tolerability, and maintenance of therapeutic effect with HP $0.01 \%$ / TAZ $0.045 \%$ lotion in the treatment of moderate-to-severe plaque psoriasis.

Keywords: Corticosteroid; Duobrii; Efficacy; Formulation; Halobetasol propionate; Lotion; Psoriasis; Retinoid; Safety; Tazarotene 


\section{Key Summary Points}

Topical therapies are widely used for the treatment for psoriasis; combination therapies utilizing more than one topical agent may provide greater efficacy, safety, and tolerability.

The corticosteroid halobetasol propionate (HP) and retinoid tazarotene (TAZ) are commonly used topical therapies that address the pathophysiology of psoriasis through complementary mechanisms of action.

A fixed-combination HP 0.01\%/TAZ $0.045 \%$ lotion was developed to simultaneously deliver the two active ingredients and moisturizing excipients in a single formulation.

HP 0.01\%/TAZ 0.045\% lotion has demonstrated improved efficacy, safety, tolerability, and maintenance of therapeutic effect compared to monotherapy with either active ingredient.

\section{DIGITAL FEATURES}

This article is published with digital features, including a summary slide, to facilitate understanding of the article. To view digital features for this article go to https://doi.org/10.6084/ m9.figshare.14662191.

\section{INTRODUCTION}

Psoriasis is a chronic inflammatory skin disorder associated with abnormal differentiation and hyperproliferation of keratinocytes, dermal infiltration of immune cells, increased vascularization, and breakdown of the epidermal barrier, resulting in raised, erythematous, scaly plaques [1-3]. Approximately $80 \%$ of patients with psoriasis have mild to moderate disease, for which topical therapy is commonly used as an integral component of treatment [4, 5]. Topical therapies are also widely used by patients with severe disease, as many do not achieve complete clearance with systemic therapies; the residual psoriasis in these patients can be managed successfully through the addition of topical therapy [6]. Among the most commonly used topical therapies for psoriasis are topical corticosteroids (TCS), vitamin D analogues, and the retinoid tazarotene (TAZ) [7]. These treatments are available in a variety of formulations and address the pathophysiology of psoriasis through anti-inflammatory, antiproliferative, immunosuppressive, and vasoconstrictive mechanisms of action [5, 8-10].

Although these topical therapies are efficacious in the treatment of psoriasis, their longterm use is limited by safety concerns. Prolonged TCS use is associated with adverse cutaneous reactions, tachyphylaxis, and rebound effects upon sudden cessation of treatment $[9,11]$; given these safety concerns, package inserts recommend no more than 2-4 weeks of continuous TCS usage [11]. TAZ has been shown to be equally efficacious as TCS while providing longer duration of effect posttreatment [12], but may be underutilized because of local irritation in lesional and perilesional skin [4]; vitamin D analogues have likewise been associated with local skin reactions [5]. Some newer treatments have combined corticosteroids with a second ingredient, such as TAZ or a vitamin D analogue, to potentially reduce adverse events (AEs) and improve efficacy [7]. For example, TAZ used together with a mid- or high-potency TCS is more effective in the treatment of psoriasis than TAZ monotherapy, is associated with fewer safety concerns than monotherapy with either active ingredient, and is recommended for the treatment of mild to moderate psoriasis [5]. Additionally, TAZ increases the duration of TCS treatment effect and the duration of psoriasis remission [5].

The need for new topical psoriasis therapies that provide improved efficacy and increased duration of remission after treatment cessation, while maintaining a favorable tolerability and safety profile, cannot be understated. In a recent 
patient survey, almost half of respondents reported being "not very" or "not at all" satisfied with their current plaque psoriasis treatment; of these, three-quarters cited a lack of effectiveness as the reason for their treatment dissatisfaction, with an additional 10\% expressing concerns about side effects and safety. Moreover, of those who had previously used a topical therapy and were currently using a biologic or systemic therapy, over half stated that they would have continued using the topical therapy had it been more effective [13]. Although the concurrent use of two topical therapies can provide improved outcomes over monotherapy, practical and technical challenges persist for therapies that include multiple active ingredients. For example, layering medications can decrease their permeability [14] and the need to apply multiple medications can complicate adherence [7].

Recently, a fixed-combination lotion containing the potent-to-superpotent corticosteroid halobetasol propionate (HP) and tazarotene (HP 0.01\%/TAZ 0.045\%; Duobrii ${ }^{\circledR}$; Ortho Dermatologics, Bridgewater, NJ, USA) was developed to meet the need for an efficacious topical psoriasis therapy with fewer safety concerns and improved duration of effect compared to monotherapy with either active ingredient [14]. This novel lotion formulation utilizes an innovative polymeric emulsion technology to provide uniform and simultaneous delivery of active ingredients at lower doses than conventional monotherapy formulations while providing enhanced hydration and moisturization.

In this review, the rationale behind the development of HP 0.01\%/TAZ 0.045\% lotion is discussed, beginning with the mechanisms of action of TAZ and HP in psoriasis. The HP $0.01 \% / \mathrm{TAZ} \quad 0.045 \%$ lotion formulation is described, and data are presented demonstrating improved penetration of the active ingredients compared to conventional formulations used alone or in layers, and benefits to epidermal barrier function. Finally, efficacy and safety data from clinical trials of HP $0.01 \% / \mathrm{TAZ}$ $0.045 \%$ lotion for the treatment of psoriasis are reviewed, highlighting how the complementary mechanisms of action of the active ingredients improve efficacy, maintenance of treatment effects, and safety. All clinical trials data presented here are from previously conducted studies; this article does not contain any new studies with human participants or animals performed by any of the authors.

\section{TAZ AND HP: MECHANISMS OF ACTION IN PSORIASIS}

\section{TAZ Mechanisms of Action}

Approved over 20 years ago, TAZ is the first topical retinoid that was developed for the treatment of psoriasis [15]. It is a retinoid prodrug that is rapidly metabolized to tazarotenic acid, which acts as a high-affinity ligand for retinoic acid receptors (RAR) $\beta$ and $\gamma$ while remaining inactive at $\operatorname{RAR} \alpha$ and retinoid $X$ receptors [15]. This receptor selectivity may contribute to the therapeutic effects of TAZ in psoriasis, as RAR $\gamma$ accounts for $90 \%$ of all RARs and is the RAR primarily found in the skin [16]. RARs serve as ligand-dependent transcription factors that can regulate the expression of psoriasis-associated genes in two ways: by binding directly to those genes' promotor sites or by modulating the activity of intermediary signaling pathways [15-17]. Through these two mechanisms, TAZ modulates keratinocyte proliferation and differentiation, downregulates pro-inflammatory mechanisms, and reduces immune cell recruitment (Fig. 1). As such, treatment with TAZ is thought to return skin to a quiescent, "prelesional" state [15].

\section{Regulation of Cell Proliferation and Differentiation}

Upon activation by tazarotenic acid, RARs directly upregulate the expression of three genes, dubbed tazarotene-induced genes (TIG1, TIG2, and TIG3). TIG1 is believed to code for a cell adhesion molecule or surface receptor that regulates cell proliferation and differentiation in a variety of tissues [18]. In a clinical study, response to TAZ treatment was associated with increased TIG1 expression in psoriatic lesional tissue [16]. TIG3 is a putative tumor suppressor 


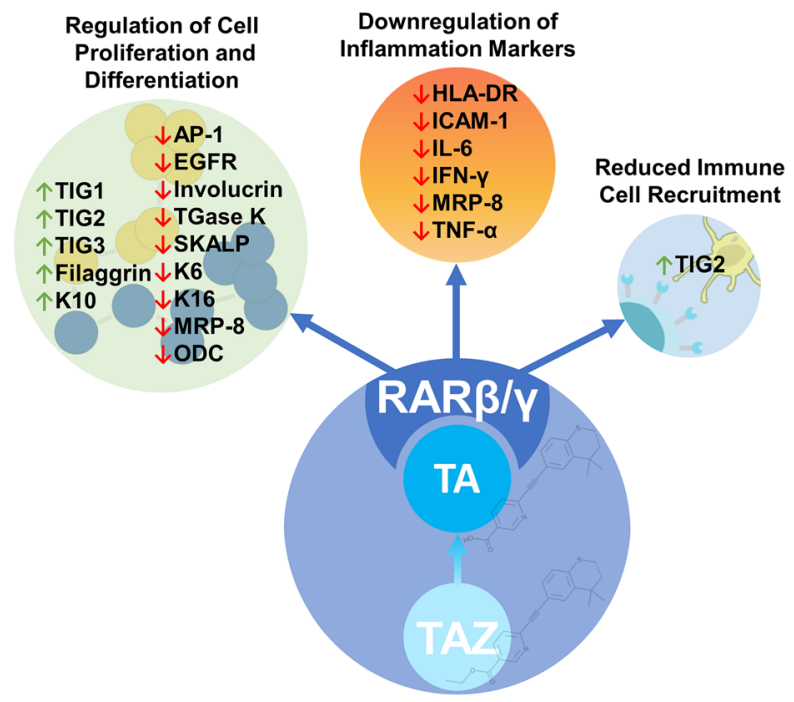

Fig. 1 Tazarotene mechanisms of action in psoriasis. TAZ is a retinoid prodrug that is rapidly metabolized to TA, which binds selectively to RAR $\beta$ and $\gamma$. Activated RARs address the pathogenic factors in psoriasis both directly (by acting as transcription factors for TIG1, 2, and 3) and indirectly (e.g., by modulating the expression or transcriptional activity of other factors, such as AP-1). AP activator protein family, EGFR epidermal growth factor receptor, HLA human lymphocyte antigen, ICAM intercellular adhesion molecule, IFN interferon, IL interleukin, K keratin, MRP migration inhibitory factor-related protein, ODC ornithine decarboxylase, RAR retinoic acid receptor, SKALP skin-derived antileukoproteinase, TA tazarotenic acid, TAZ tazarotene, TGase $\mathrm{K}$ keratinocyte transglutaminase, TIG tazarotene-induced gene, TNF tumor necrosis factor

that also appears to be particularly important for the terminal differentiation of keratinocytes [19]; its activity is downregulated in epidermal hyperproliferative diseases such as psoriasis and skin cancer [20]. Though TIG2 may have antiproliferative effects [16], it also contributes to immune cell recruitment and inflammatory processes (discussed below). In psoriatic lesional tissue, expression of all three TIGs is low, and is upregulated by treatment with TAZ [16].

In addition to directly modulating gene expression, TAZ-induced RAR activation can indirectly modulate processes underlying keratinocyte proliferation and differentiation in psoriatic lesions [16, 21]. TAZ-activated RARs inhibit dimerization of proteins in the activator protein 1 (AP-1) family of transcription factor complexes [22]. Blocking dimerization prevents AP-1 from driving the expression of genes for many cytokines-including tumor necrosis factor alpha (TNF $\alpha)$, interleukin (IL)-4, and interferon- $\gamma$ (IFN $\gamma)$-that are secreted by activated immune cells and infiltrating T cells and that promote keratinocyte hyperproliferation [23-26]. In patients with psoriasis, genes enriched in lesional versus nonlesional tissue were associated with AP-1 binding sites and cytokine induction [27]. In preclinical findings, TAZ antagonized AP-1-dependent gene expression [28]; such downregulation of AP-1 activity has been associated with amelioration of psoriasislike lesions [29]. In addition to its effects on cytokine production, TAZ antagonizes AP-1mediated expression of ornithine decarboxylase, a key regulator of the synthesis of polyamines that are required for cell proliferation [28].

TAZ-activated RARs can also normalize the expression of epidermal growth factor receptors (EGFR), which directly stimulate growth and differentiation in many epithelial tissues and are overexpressed in psoriatic perilesional skin [30]. Alterations in EGFR cause abnormal cell proliferation and may cause human skin disease $[30,31]$. Conversely, reduction in EGFR is one of the first detectable changes that occurs with many treatments for psoriasis; these receptors begin to disappear as the skin normalizes and parakeratosis begins to resolve [30]. In patients with psoriasis, 2 weeks of TAZ treatment was associated with reduced EGFR expression [32].

TAZ has demonstrated the ability to reverse over- and underexpression of many other markers of keratinocyte differentiation and cell proliferation in psoriatic lesional tissue, including: filaggrin, involucrin, and keratins (e.g., K6, 10, and 16), which are structural proteins underlying the cornified envelope; migration inhibitory factor-related protein 8 (MRP-8, aka protein $\mathrm{S100-A8),} \mathrm{which} \mathrm{binds} \mathrm{to} \mathrm{keratin}$ filaments and associates with the epidermal cytoskeleton; keratinocyte transglutaminase type I (TGase K), which catalyzes the assembly of the cornified envelope; and skin-derived antileukoproteinase (SKALP), a protease/elastase 
inhibitor (reviewed in Duvic et al., 1997; 1998; Lee, 2020) [16, 21, 33].

\section{Downregulation of Inflammation Markers and Reduced Immune Cell Recruitment}

Psoriatic lesions arise in part from immune cells, local skin cells, and cytokines interacting to create a pro-inflammatory environment that promotes keratinocyte proliferation. In keratinocytes, TNF $\alpha$ and IFN $\gamma$ induce the expression of intracellular adhesion molecule-1 (ICAM-1); IFN $\gamma$ also induces expression of human lymphocyte antigens DR (HLA-DR) [34]. ICAM-1 expression is particularly correlated with the degree of dermal inflammation [34], whereas HLA-DR expression correlates with psoriasis severity [21]. These cell surface antigens are increased in untreated psoriatic skin compared to nonlesional or normal skin and may recruit $T$ cells to the epidermis in psoriasis $[16,21,34]$. In clinical studies, TAZ treatment decreased levels of ICAM-1 and HLA-DR in patients with psoriasis [32] and decreased dermal infiltration of leukocytes (e.g., CD45 ${ }^{+}$ $\mathrm{T}$ cells) in patients with $\mathrm{T}$ cell lymphoma [35]. As noted above, TAZ downregulates the expression of MRP-8 [16], the production and secretion of which is stimulated by TNFa. In addition to its role in cell proliferation/differentiation, MRP-8 recruits leukocytes to inflammation sites, further stimulating the expression of $\mathrm{TNF} \alpha$, IL- 6 , and other pro-inflammatory cytokines-thus creating a positive feedback loop for epidermal inflammation [36, 37].

Additionally, TAZ regulates immune responses and inflammation in psoriasis by upregulating the expression of TIG2, which is hypothesized to play a role in maintaining the normal physiology of skin $[16,38]$. The protein product of TIG2, chemerin, plays a complex role in immune and inflammatory processes. In early stages of inflammation, chemerin coordinates pro-inflammatory immune responses such as the trafficking of plasmacytoid dendritic cells (DCs) to the site of inflammation $[39,40]$. These DCs are among the most abundant sources of the cytokine IFN $\alpha$, which stimulates the maturation of immune cells and the production of many other cytokines; thus, activation of DCs may represent an early step in psoriasis pathogenesis [41]. Consistent with this, chemerin levels are elevated in new, evolving psoriatic plaques and in pre-lesional dermis relative to normal or uninvolved skin [41]. In contrast, at the end of inflammation, chemerin transforms to play an anti-inflammatory role [40]. Consequently, in chronic, non-evolving psoriatic lesions, chemerin expression in the epidermis may favor the clearance of immune cells from inflamed tissue and the restoration of skin homeostasis [42]. Thus, upregulation of TIG2 expression in chronic psoriatic lesions by TAZ may support the anti-immune and anti-inflammatory mechanisms of chemerin.

\section{HP Mechanisms of Action}

Corticosteroids such as HP have been used in dermatology for over 50 years and their physiological effects have been reviewed extensively $[9,43,44]$. They impact the pathophysiology of psoriasis via activation of glucocorticoid

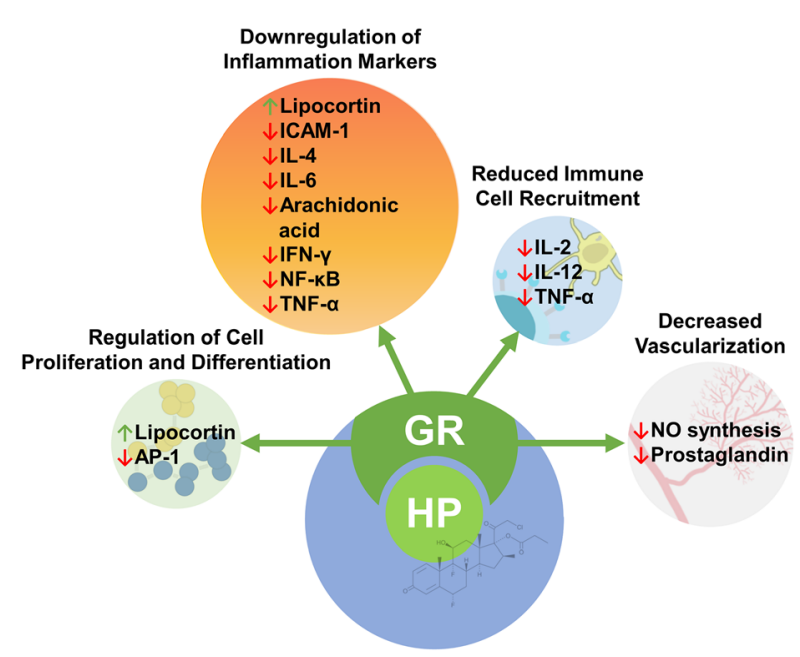

Fig. 2 Halobetasol propionate mechanisms of action in psoriasis. HP is a synthetic glucocorticoid that binds directly to GR. Activated GR can exert both genomic (e.g., upregulation of lipocortin synthesis) and non-genomic (e.g., suppression of pro-inflammatory cytokines) effects to address the pathophysiology of psoriasis. AP activator protein, GR glucocorticoid receptor, HP halobetasol propionate, ICAM intercellular adhesion molecule, IL interleukin, IFN interferon, NF- $\mathrm{KB}$ nuclear factor kappalight-chain-enhancer of activated $\mathrm{B}$ cells, $\mathrm{NO}$ nitric oxide, TNF tumor necrosis factor 
receptors (GR) which, like activated RARs, exert both genomic and non-genomic actions to regulate transcription of genes for anti-inflammatory and pro-inflammatory mediators (e.g., IL-6, NF- $\kappa B$, and TNF $\alpha$; Fig. 2) [43]. HP-induced GR activation decreases activity of AP-1 proteins, though through a different pathway than TAZ-induced RARs; whereas activated RARs prevent the dimerization of AP-1 proteins, activated GRs reduce their phosphorylation [9]. GR-mediated stimulation of lipocortin synthesis leads to decreased conversion of cell membrane phospholipids into pro-inflammatory arachidonic acid [45]. In addition, activation of GRs inhibits the expression and activity of many other cytokines and chemokines that traffic pro-inflammatory mediators to the site of inflammation (reviewed in Barnes 1998; Uva et al. 2012) [9, 43].

Corticosteroids also mitigate vascular, cell proliferation, and immunological features of psoriasis, though the mechanisms underlying these effects are not as well understood. GR activation inhibits prostaglandin formation and mitigates inflammation-induced nitric oxide synthesis [46, 47], thus causing vasoconstriction and reducing the delivery of pro-inflammatory mediators to areas treated with TCS [9]. However, tachyphylaxis to prolonged TCS use may occur as a result of the loss of vasoconstriction at the level of capillaries [48]. In addition to effects on inflammation, lipocortin upregulation by corticosteroids may regulate keratinocyte hyperproliferation by inhibiting epidermal mitosis [9]. Corticosteroids also repress the maturation, differentiation, and proliferation of immune cells, leading to reduced production of pro-inflammatory mediators [9]. The antiproliferative mechanisms of corticosteroids may contribute to their atrophogenic potential. In addition to reduced keratinocyte proliferation [9], fibroblast proliferation is suppressed, and there is decreased production of mucopolysaccharides (e.g., hyaluronic acid), elastin, matrix metalloproteases, and collagen 1 and 3. Mast cell numbers are reduced, dermal support is lost, and skin becomes thinned and loses elasticity [49].

\section{HP $0.01 \% / T A Z ~ 0.045 \%$ LOTION FORMULATION}

\section{Benefits of Concurrent Use of TAZ and TCS}

Psoriasis therapies employing both TAZ and a TCS have demonstrated improved efficacy over monotherapy with either treatment. In one of the first studies to evaluate the efficacy of TAZ used in combination with a TCS, the time to initial treatment success was reduced from 4 weeks with TAZ monotherapy to 2 weeks with TAZ plus mometasone furoate. Moreover, the percentages of participants achieving treatment success and reductions in body surface area (BSA) involvement were significantly greater with the combination therapy [50]. Similarly, a separate study found that TAZ plus mometasone furoate yielded greater and more rapid improvements in psoriasis than mometasone furoate monotherapy. Moreover, combination therapy yielded greater duration of posttreatment maintenance of effects and decreased discontinuations due to posttreatment disease recurrence [51]. The enhanced efficacy of TAZ and TCS when used together may reflect their complementary mechanisms of action, including distinct processes by which they regulate hyperproliferation (e.g., filaggrin upregulation by TAZ and lipocortin upregulation by TCS) and inflammation (e.g., non-overlapping mechanisms of AP-1 inhibition). Posttreatment maintenance of therapeutic effect may be due to normalization of gene expression in lesional skin by TAZ.

When used together, TAZ and TCS can also mitigate the safety and tolerability concerns associated with use of either as monotherapy, as evidenced by low incidences of AEs and steroid rebound in clinical trials [52]. Safety concerns with TAZ include application site pain, itching, and swelling; in preclinical studies, these effects were blocked by antagonism or ablation of the irritant receptor TRPV1, suggesting a mechanism underlying these side effects [53]. GR activation enhances the degradation of TRPV1 [54], thus providing a means by which TCS may mitigate TAZ-induced irritation. Erythema, another common local skin reaction to TAZ, 
may be lessened by the vasoconstrictive effects of TCS. One of the greatest concerns with prolonged TCS use is skin atrophy, caused in part by decreased collagen synthesis and inhibition of cell proliferation [44]. In treatment of psoriatic perilesional skin, addition of a retinoid to TCS therapy partially protected against TCS-induced epidermal atrophy [55]. TAZ has been shown to increase epidermal thickness in photodamaged skin [56] and to reduce TCS-induced atrophy in healthy skin [57]. Thus, combination of TAZ with TCS may mitigate the risk of steroid-induced atrophy.

Given the benefits to efficacy and safety with concurrent use of TCS and TAZ, a fixed-combination HP $0.01 \% / \mathrm{TAZ} \quad 0.045 \%$ lotion was developed to leverage the beneficial properties of both active ingredients, while mitigating safety concerns, in a single formulation. The following sections describe the properties of this unique formulation and review clinical trials of HP $0.01 \% / T A Z ~ 0.045 \%$ lotion in the treatment of psoriasis, with a focus on synergistic efficacy of HP and TAZ and posttreatment maintenance of treatment effects.

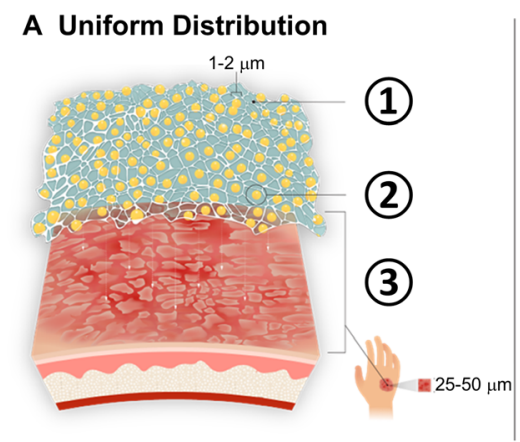

B Mesh Dissolves

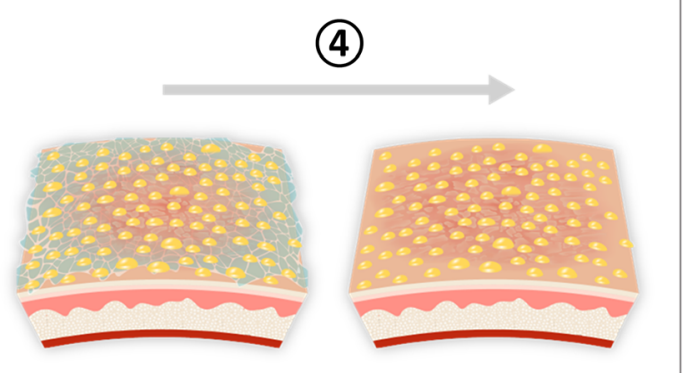

\section{Polymeric Emulsion-Based Vehicle Technology}

The vehicle used to deliver a topical therapy can significantly impact the drug's pharmacokinetics, efficacy, and safety profile [58], as well as patient adherence to treatment [59]. Thus, in dermatology, optimization of the topically applied vehicle represents an opportunity to maximize therapeutic benefits. The novel formulation for fixed-combination HP 0.01\%/TAZ $0.045 \%$ lotion utilizes polymeric emulsion technology that allows active ingredients and moisturizing excipients to be encapsulated together within the same oil droplets (Fig. 3) [14]. These droplets are evenly distributed throughout a three-dimensional mesh matrix, which breaks apart upon contact with salts on the skin to provide more rapid and efficient delivery of moisturizers, hydrating agents, and active ingredients into dermal layers than with the individual ingredients applied either alone or in layers.

\section{Improved Delivery of Active Ingredients}

The improved delivery of active ingredients with this fixed-combination lotion was demonstrated in two percutaneous permeation
Fig. 3 Fixed-combination HP 0.01\%/TAZ 0.045\% lotion formulation. HP $0.01 \% /$ TAZ $0.045 \%$ lotion was developed using novel polymeric emulsion technology to distribute active ingredients and excipients rapidly, efficiently, and evenly across the skin. (1) 3-D mesh (polymeric matrix) holds water and water-soluble hydrating agents. (2) Droplets $(1-2 \mu \mathrm{m})$ consisting of active ingredients and oil-soluble moisturizing agents held apart by the 3-D mesh. (3) 3-D mesh allows for uniform distribution of active ingredients and moisturizing agents. (4) 3-D mesh dissolves instantly upon contact with salts on skin. (5) Active ingredients and moisturizing/hydrating agents uniformly absorbed by skin. Figure (c) 2021 Ortho Dermatologics' affiliated entities. Adapted with permission. HP halobetasol propionate, TAZ tazarotene 
studies [14]. HP 0.01\%/TAZ 0.045\% lotion demonstrated higher permeation efficiency of $\mathrm{HP}$, with $6.8 \%$ of the applied dose observed in dermal levels at $24 \mathrm{~h}$. In comparison, only 3.3\% of the applied dose of HP $0.05 \%$ cream was observed, despite having five times greater concentration of HP. Similarly, HP 0.01\%/TAZ $0.09 \%$ lotion yielded higher percutaneous absorption of TAZ, with dermal levels of $20.8 \%$ of the applied dose at $24 \mathrm{~h}$ versus $12.3 \%$ with TAZ $0.1 \%$ cream alone [14]. In contrast, when TAZ $0.1 \%$ cream was layered on top of HP $0.05 \%$ cream, the permeability of TAZ was decreased [14]. Moreover, a fixed combination cream formulation of TAZ plus the TCS betamethasone dipropionate showed no improvement in the percutaneous absorption of either active ingredient relative to its monads [60]. Thus, the improved permeation efficiency with HP $0.01 \% / \mathrm{TAZ} 0.045 \%$ lotion may be unique to this polymeric emulsion-based fixedcombination lotion formulation.

\section{Improved Epidermal Barrier Function}

A common feature of psoriasis is loss of epidermal barrier function [3, 61]. Topical treatment with both TCS and TAZ is associated with adverse effects on the integrity of the stratum corneum, thus potentially exacerbating the effect of psoriasis on epidermal barrier functions $[62,63]$. Within a few weeks of treatment initiation with TAZ or other topical retinoids, patients often experience "retinoid dermatitis," characterized by erythema, scaling, itching/ burning, and desquamation due to loss of the stratum corneum $[62,64]$. Prolonged TCS usage may decrease lipid synthesis in the stratum corneum, thus delaying repair of epidermal barrier damage due to psoriasis and expediting the return of disease after cessation of TCS treatment [63]. Reduction in stratum corneum barrier function increases transepidermal water loss (TEWL); this can be mitigated by adjunctive barrier repair therapies such as moisturizers $[62,63,65]$.

The vehicle formulation for HP $0.01 \% / \mathrm{TAZ}$ $0.045 \%$ lotion-developed to provide enhanced barrier to the skin-delivers a number of excipients to augment skin moisturization and mitigate potential adverse effects of the active ingredients. Oil droplets, humectants, and emollients work in concert to attract and retain water content within the stratum corneum while simultaneously providing an occlusive barrier to prevent moisture loss [14]. In 30 healthy female participants, vehicle lotion increased skin moisture content (assessed via corneometry) and improved skin barrier function (assessed via TEWL) relative to untreated skin. Significant improvements in corneometry and TEWL were observed 15 min post-application and persisted through $24 \mathrm{~h}$ of follow-up. Specifically, mean corneometry scores peaked at 15-30 min post-application, with a more than twofold increase (improvement) from baseline; mean TEWL scores decreased (improved) from baseline by approximately half at $8 \mathrm{~h}$ post-application $[13,14]$. Together, these data demonstrate that HP $0.01 \% / \mathrm{TAZ} \quad 0.045 \%$ lotion provides rapid and sustained increases in skin moisturization and decreases in water loss over $24 \mathrm{~h}$.

\section{Patient Acceptance}

Vehicle formulation impacts not only drug delivery but also patient acceptance and adherence to treatment. Patients generally prefer vehicles that are lighter, non-greasy, and easy to use $[66,67]$. These cosmetic characteristics can significantly impact patient acceptance, treatment adherence, real-world effectiveness, and quality of life $[14,59,68]$. In a patient perception evaluation of HP $0.01 \% / \mathrm{TAZ}$ $0.045 \%$ lotion vehicle, acceptability of the vehicle lotion was very high, with $93-100 \%$ responding favorably to questions regarding its application, feel, aesthetics, and moisturizing/ hydrating properties [14]. Ease of use with this lotion is supported by the simultaneous delivery of active ingredients and moisturizers/hydrating agents in one formulation, thus reducing the need to apply moisturizers separately. Further, the polymeric emulsion lotion formulation has demonstrated increased spreadability compared to a retinoid cream formulation [69]. Thus, HP $0.01 \% /$ TAZ $0.045 \%$ lotion provides efficient delivery of active ingredients in an easy-to-spread lotion formulation that is aesthetically pleasing to patients. 
Table 1 Clinical trials of HP 0.01\%/TAZ 0.045\% lotion

\begin{tabular}{|c|c|c|c|}
\hline Study & Phase $2[13,70]$ & Pooled phase $3^{a}[71]$ & Long-term phase 3 [73] \\
\hline Study design & Randomized; double-blind & Randomized; double-blind & Open-label \\
\hline Treatment duration & $\begin{array}{l}8 \text { weeks of once-daily } \\
\text { treatment }\end{array}$ & $\begin{array}{l}8 \text { weeks of once-daily } \\
\text { treatment }\end{array}$ & $\begin{array}{l}8 \text { weeks of once-daily treatment, } \\
\text { followed by treatment in } \\
\text { 4-week cycles as needed }\end{array}$ \\
\hline Follow-up & $\begin{array}{l}\text { Week } 12 \text { (4 weeks } \\
\text { posttreatment) }\end{array}$ & $\begin{array}{l}\text { Week } 12 \text { (4 weeks } \\
\text { posttreatment) }\end{array}$ & Every 4 weeks until week 52 \\
\hline \multirow[t]{4}{*}{ Treatment arms $\left(N^{b}\right)$} & HP/TAZ (59) & $\mathrm{HP} / \mathrm{TAZ}(276)$ & $\mathrm{HP} / \mathrm{TAZ}(555)$ \\
\hline & $\mathrm{HP}(63)$ & Vehicle (142) & \\
\hline & TAZ (59) & & \\
\hline & Vehicle (31) & & \\
\hline
\end{tabular}

Inclusion in all studies required an IGA score of 3 (moderate) or 4 (severe) and affected BSA of 3-12\%. In all studies, active ingredients were delivered in the same lotion vehicle

$B S A$ body surface area, $H P$ halobetasol propionate $0.01 \%$ lotion, $H P / T A Z$ halobetasol propionate $0.01 \%$ and tazarotene 0.045\% lotion, IGA Investigator's Global Assessment, $T A Z$ tazarotene $0.045 \%$ lotion

${ }^{a}$ Data pooled from two studies

${ }^{\mathrm{b}}$ For phase 2 and pooled phase 3 studies, $N$ values represent number of participants in the intent-to-treat population. For long-term phase 3 study, $N$ value represents all participants treated with HP/TAZ lotion

${ }^{c}$ All participants were treated with HP/TAZ lotion once daily for 8 weeks. At week 8 , treatment was stopped for participants who achieved treatment success (IGA $0=$ clear or $1=$ almost clear); all other participants were treated for an additional 4 weeks). All participants were re-evaluated at week 12 for improvement ( $\geq 1$ point decrease from baseline IGA score). Participants demonstrating improvement continued in the study and were managed in 4-week cycles for up to 1 year. Participants were evaluated every 4 weeks for treatment success; those achieving success stopped treatment for 4 weeks; those not experiencing success continued once-daily HP/TAZ lotion for 4 weeks. Maximum continuous exposure allowed was 24 weeks

\section{HP 0.01\%/TAZ 0.045\% LOTION: EFFICACY AND SAFETY IN THE TREATMENT OF PSORIASIS}

\section{Efficacy of HP 0.01\%/TAZ 0.045\% Lotion}

The efficacy and safety of HP $0.01 \% /$ TAZ $0.045 \%$ lotion have been demonstrated in one phase 2 and two phase 3 randomized, doubleblind clinical trials, and one long-term (52week) open-label phase 3 study (Table 1). In the phase 2 study, rates of treatment success were greater for HP $0.01 \% / \mathrm{TAZ} 0.045 \%$ lotion versus HP $0.01 \%$ and TAZ $0.045 \%$ alone after 8 weeks of once-daily treatment (Fig. 4); note that both monads were delivered in the same lotion vehicle as the fixed-combination treatment
[70]. This efficacy was confirmed in data pooled from the larger phase 3 studies and in the longterm open-label study [71-73]. HP 0.01\%/TAZ $0.045 \%$ lotion was also superior to its monads and to vehicle alone in reducing psoriasis signs of erythema, plaque elevation, and scaling and reducing affected BSA [70-72]. Importantly, post hoc analyses demonstrated that the superiority of HP $0.01 \% / \mathrm{TAZ} \quad 0.045 \%$ lotion to vehicle was consistent across many subgroups of participants in the phase 3 studies, including male and female subjects [74] and participants with skin of color [75], target lesions on the lower extremities [76], or lower BSA involvement (3-5\%) at baseline [77]. 


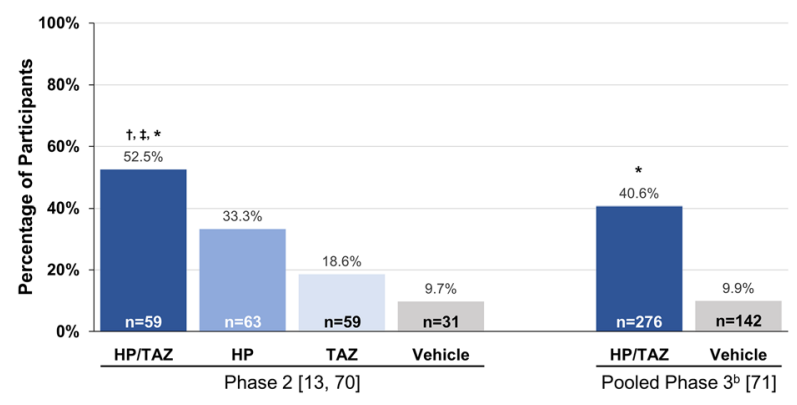

Fig. 4 Treatment success ${ }^{\mathrm{a}}$ after 8 weeks of once-daily treatment with HP $0.01 \% / \mathrm{TAZ} 0.045 \%$ lotion (placebocontrolled trials). ${ }^{\dagger} P<0.05$ vs HP; ${ }^{\ddagger} P<0.001$ vs TAZ; ${ }^{*} P<0.001$ vs vehicle. ${ }^{a}$ Treatment success was defined as at least a two-grade reduction in IGA score and a score of 0 (clear) or 1 (almost clear). ${ }^{\mathrm{b}}$ Data pooled from two studies. $N$ values represent total number of participants in the intent-to-treat population for each study. All active ingredients were delivered in the same lotion vehicle. HP halobetasol propionate $0.01 \%$ lotion, $\mathrm{HP} / \mathrm{TAZ}$ halobetasol propionate $0.01 \%$ and tazarotene $0.045 \%$ lotion, IGA Investigator's Global Assessment, TAZ tazarotene 0.045\% lotion

\section{Safety and Tolerability of HP $0.01 \% / \mathrm{TAZ}$ $\mathbf{0 . 0 4 5 \%}$ Lotion}

In the phase 2 and phase 3 clinical trials and in the long-term open-label study, HP 0.01\%/TAZ $0.045 \%$ lotion demonstrated a favorable safety profile and was well tolerated (Table 2) [70-72, 78]. The majority of AEs reported were of mild or moderate severity, and the most common treatment-related AEs were (contact) dermatitis, (application site) pruritus, and application site pain. In the pooled phase 3 studies, greater reductions in the severity of itching, dryness, and burning/stinging skin symptoms were achieved with HP 0.01\%/TAZ $0.045 \%$ than vehicle after 8 weeks of treatment [72].

Importantly, HP $0.01 \% /$ TAZ $0.045 \%$ lotion is associated with low incidence of AEs that are of greatest concern with HP and TAZ monotherapy. Skin atrophy is one of the most problematic adverse side effects associated with prolonged TCS use, occurring in up to $5 \%$ of participants in clinical trials of TCS treatment for psoriasis [79]. Because TCS therapy is limited to $2-4$ weeks [11], there is little data on the incidence of atrophy with longer-term use. In the phase 2 and pooled phase 3 clinical trials, during which participants received 8 weeks of once-daily HP $0.01 \% / T A Z$ 0.045\% lotion, incidences of atrophy were rare and generally resolved by study end [70, 71]. In the long-term open label study, participants received up to 24 weeks of continuous treatment with HP $0.01 \% /$ TAZ $0.045 \%$ lotion. Despite this prolonged exposure, peak incidence of atrophy was low $(2.3 \%$ at week 8$)$ and declined through study end. Atrophy was reported as an AE in only four participants $(0.7 \%)$ and led to only one discontinuation [73]. This low rate of atrophy-even with up to 24 weeks of exposure to HP $0.01 \% / T A Z ~ 0.045 \%$ lotion-may reflect the mechanism of action of TAZ, as retinoids have been shown to increase dermal fibroblast number and activity as well as increase production of collagen and elastin $[80,81]$.

Irritation-related AEs with TAZ are of concern to both patients and clinicians, and likely cause some avoidance of TAZ monotherapy for psoriasis [8]. HP $0.01 \% / \mathrm{TAZ} 0.045 \%$ lotion is associated with lower rates of application-site AEs relative to TAZ monotherapy, perhaps owing to the anti-inflammatory actions of HP [8]. In the phase 2 study, treatment-emergent AEs (TEAEs) were less frequent through week 8 for HP $0.01 \% /$ TAZ $0.045 \%$ lotion (33.9\%) than for TAZ alone (46.6\%), as were discontinuations from the study due to an $\mathrm{AE}(3.4 \%$ versus 12.1\%; Table 2) [70]. Similar rates of TEAEs and discontinuations were observed in the pooled phase 3 studies (35.9\% and 6.3\%, respectively) [71]. In the phase 3 studies, the most commonly reported treatment-related AEs through week 8 with HP $0.01 \% /$ TAZ $0.045 \%$ lotion were contact dermatitis $(6.3 \%)$, application site pain $(2.6 \%)$, and pruritus $(2.2 \%)$; few or no participants reported erythema $(n=0)$ or skin irritation $(n=2)$ as treatment-related AEs [71]. With longer-term treatment in the open-label study, rates of these AEs decreased to less than $1 \%$ by week 36 [73, 78]. In comparison, across six clinical trials of TAZ monotherapy $(0.1 \%$ or $0.05 \%$; cream or gel formulations), rates were similar for contact dermatitis (3-8\%) but considerably greater for application site pain 
Table 2 Safety of HP 0.01\%/TAZ 0.045\% lotion

\begin{tabular}{|c|c|c|c|c|c|c|c|}
\hline \multirow{2}{*}{$\begin{array}{l}\text { Study } \\
\text { Treatment arms }\end{array}$} & \multicolumn{4}{|c|}{ Phase $2[13,70]$ (through week 8 ) } & \multicolumn{2}{|c|}{$\begin{array}{l}\text { Pooled phase } 3^{a}[13,71] \\
\text { (through week } 8 \text { ) }\end{array}$} & \multirow{2}{*}{$\begin{array}{l}\begin{array}{l}\text { Long-term open- } \\
\text { label }[13,73] \\
\text { (through week 12) }\end{array} \\
\text { HP/TAZ } \\
\quad(n=527)\end{array}$} \\
\hline & $\begin{array}{l}\mathrm{HP} / \mathrm{TAZ} \\
\quad(n=59)\end{array}$ & $\begin{array}{l}\mathrm{HP} \\
\quad(n=62)\end{array}$ & $\begin{array}{l}\text { TAZ } \\
\quad(n=58)\end{array}$ & $\begin{array}{l}\text { Vehicle } \\
\qquad(n=31)\end{array}$ & $\begin{array}{l}\mathrm{HP} / \mathrm{TAZ} \\
\quad(n=270)\end{array}$ & $\begin{array}{l}\text { Vehicle } \\
\qquad(n=140)\end{array}$ & \\
\hline$\geq 1$ TEAE, $\%$ & 33.9 & 21.0 & 46.6 & 22.6 & 35.9 & 21.4 & 42.3 \\
\hline $\begin{array}{l}\text { Discontinuations } \\
\text { due to AE, } \\
n(\%)\end{array}$ & $2(3.4)$ & 0 & $7(12.1)$ & $1(3.2)$ & $17(6.3)$ & $5(3.6)$ & $30(5.7)$ \\
\hline \multicolumn{8}{|c|}{ Common treatment-related TEAEs ( $\geq 2 \%$ in any active treatment arm), $n(\%)$} \\
\hline $\begin{array}{l}\text { Application site } \\
\text { pain }\end{array}$ & $2(3.4)$ & 0 & $5(8.6)$ & $1(3.2)$ & $7(2.6)$ & $1(0.7)$ & $24(4.6)$ \\
\hline Dermatitis $^{c, d}$ & 0 & 0 & $1(1.7)$ & 0 & $17(6.3)$ & 0 & $38(7.2)$ \\
\hline Pruritus $^{c}$ & 0 & 0 & $4(6.9)$ & 0 & $6(2.2)$ & $4(2.9)$ & $22(4.2)$ \\
\hline Erythema $^{c}$ & $1(1.7)$ & 0 & $2(3.4)$ & 0 & 0 & 0 & $5(0.9)$ \\
\hline Folliculitis $^{c}$ & $2(3.4)$ & 0 & 0 & 0 & $5(1.9)$ & $1(0.7)$ & $9(1.7)$ \\
\hline
\end{tabular}

For long-term open-label study, safety data through week 52 have been published [73, 78]

In all studies, active ingredients were delivered in the same lotion vehicle

$A E$ adverse event, $H P$ halobetasol propionate $0.01 \%$ lotion, $H P / T A Z$ halobetasol propionate $0.01 \%$ and tazarotene $0.045 \%$ lotion, $T A Z$ tazarotene $0.045 \%$ lotion, TEAE treatement-emergent adverse event

a Data pooled from two studies

b $N$ values represent number of participants in the safety population

c Application site TEAEs in phase 2 and long-term open-label studies

d Contact dermatitis in pooled phase 3 studies

(4-11\%), pruritus (11-29\%), and erythema (2-28\%) [82].

When participants were stratified by baseline disease severity, tolerability of HP 0.01\%/TAZ $0.045 \%$ lotion was greatest among participants with moderate disease (IGA score of $3, n=232$ ). Compared to participants with severe disease at baseline (IGA score of $4, n=38$ ), moderate IGA participants experienced lower treatment-related TEAE rates of application site pain $(1.7 \%$ vs $7.9 \%)$ and pruritus ( $1.7 \%$ vs $5.3 \%)$, though rates of contact dermatitis were comparable (moderate, 6.5\%; severe, 5.3\%) [13]. A similar pattern was observed when stratifying participants by severity of skin symptoms (burning/ stinging, dryness, and itching). HP 0.01\%/TAZ $0.045 \%$-treated participants with moderate/severe symptoms at baseline (severity score of 2 or 3 on a scale of 0 [none] to 3 [severe]) were approximately two times more likely than participants with less severe symptoms at baseline (severity score of 0 [none] or 1 [mild]) to have moderate/severe symptoms at any postbaseline timepoint [13].

A limitation in interpretation of safety data from the clinical trials is that unlike real-world use, participants were required to apply HP $0.01 \% /$ TAZ $0.045 \%$ lotion once daily for eight continuous weeks, regardless of treatment response [71, 72]. As such, it is possible that 
continuing treatment beyond achievement of treatment success or clearance of psoriasis may have contributed to irritation. In the clinic, patient education and a nuanced approach to treatment may help ameliorate irritation-related AEs, particularly because the prescribing information for HP $0.01 \% / \mathrm{TAZ} 0.045 \%$ lotion does not provide a minimum or maximum duration of continuous treatment [83]. Clinicians may want to educate patients on the importance of minimizing treatment after achievement of clear skin and avoiding the overuse of HP $0.01 \% / \mathrm{TAZ} 0.045 \%$ lotion. For individuals experiencing irritation, it is the authors' opinion that patients should be advised to temporarily interrupt drug use (i.e., drug holiday) until irritation signs and symptoms have subsided. The authors also recommend encouraging the use of moisturizerswhich are recommended for use with TCS [5]concurrent with treatment and/or during periods of irritation-related treatment cessation, to enhance epidermal barrier function.

\section{Synergistic Efficacy of HP and TAZ}

Concurrent use of two complementary active ingredients often provides a cumulative or additive effect. In rarer cases, a synergistic effect is seen wherein the efficacy of the combination is greater than the summed efficacy of the individual ingredients. In the phase 2 study, the treatment success rate of HP 0.01\%/TAZ 0.045\% lotion was measured against HP alone, TAZ alone, and vehicle. Thus, relative efficacy could be assessed using the ratio of the benefit of HP $0.01 \% / \mathrm{TAZ} 0.045 \%$ divided by the summed benefits of HP and TAZ alone; synergy of HP $0.01 \% / \mathrm{TAZ} 0.045 \%$ lotion would thus be demonstrated by a ratio greater than 1.0 [84]. A synergistic effect on treatment success was observed as early as week 2 , at which point treatment benefit of HP $0.01 \% / \mathrm{TAZ} \quad 0.045 \%$ lotion was $11.9 \%$, compared to $6.5 \%$ for $\mathrm{HP}+$ TAZ (ratio of 1.8) [85]. This effect continued through week 8 of treatment (HP $0.01 \% / \mathrm{TAZ}$ 0.045\%: 42.8\%; HP + TAZ: $32.5 \%$; ratio of 1.3 ) and 4 weeks posttreatment (ratio of 1.6). A similar pattern was observed for reductions from baseline BSA [85]. This apparent synergy of HP $0.01 \% / T A Z ~ 0.045 \%$ lotion could result from the combination of the complementary antiinflammatory and antiproliferative mechanisms of HP and TAZ. Notably, a fixed-combination formulation of the vitamin $\mathrm{D}$ analogue calcipotriene plus the mid-strength corticosteroid betamethasone dipropionate (Cal/BD), though also more efficacious than its monads [5], has not demonstrated synergistic efficacy beyond the additive effects of the individual active ingredients.

\section{Maintenance of Effect After Treatment Cessation}

Among the benefits of TAZ in psoriasis therapy is a prolonged duration of remission after treatment cessation. In a clinical trial of TAZ monotherapy, significantly greater clinical success rate for TAZ versus vehicle was maintained for 12 weeks after cessation of TAZ treatment [86]. This maintenance of effect was also observed in a clinical trial of TAZ used in conjunction with a TCS. After 12 weeks of treatment, TAZ plus TCS was superior to TCS alone in global improvements as well as individual signs of psoriasis, and this superiority was maintained throughout a 12-week posttreatment follow-up period [51]. This prolongation of therapeutic effect may be due to TAZ returning skin to a normalized, "pre-lesional" state [15]. In clinical trials of HP $0.01 \% / \mathrm{TAZ}$ $0.045 \%$ lotion, the majority of participants who achieved treatment success also experienced prolonged maintenance of the therapeutic effect after treatment cessation. In the pooled phase 3 clinical trials, $63 \%$ of participants who achieved treatment success at week 8 remained treatment successes 4 weeks posttreatment, and three-quarters of participants maintained or improved their reductions in affected BSA [87].

Consistent with observations from these studies, the majority of participants in the longterm open-label study experienced durable therapeutic effects after cessation of HP $0.01 \%$ / TAZ $0.045 \%$ treatment. In this 1-year study, maintenance of treatment success endured for over 1 month in approximately $55 \%$ of 


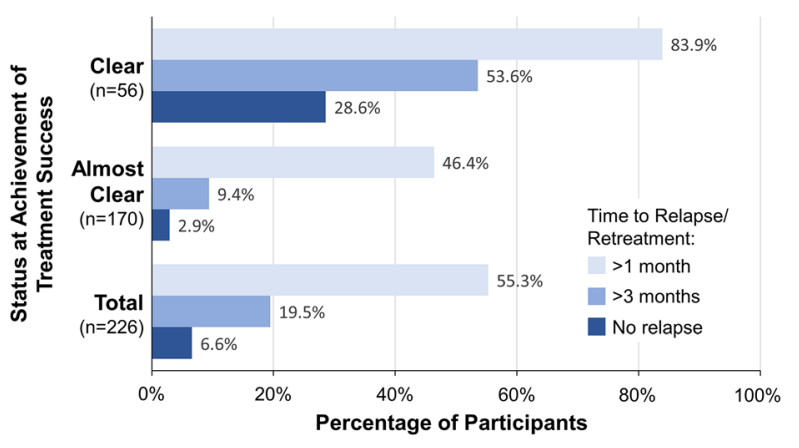

Fig. 5 Maintenance of treatment success with HP 0.01\%/ TAZ $0.045 \%$ lotion (open-label long-term phase 3 study $[73,88])$. Data shown for participants enrolled post 8 weeks in the study and who stopped therapy after achieving treatment success, defined as an IGA score of 0 (clear) or 1 (almost clear). Cumulative data shown. "> 3 months" includes participants who did not relapse or require any retreatment for the duration of the study; "> 1 month" includes participants who did not require retreatment for $>3$ months or who did not relapse or require any retreatment for the duration of the study. HP/ TAZ halobetasol propionate $0.01 \%$ and tazarotene 0.045\%, IGA Investigator's Global Assessment

participants who stopped treatment after achieving treatment success, and $6.6 \%$ did not experience any posttreatment relapse for the duration of the study (Fig. 5) [73]. One important consideration with this finding is that participants were required to stop treatment upon achievement of treatment success, defined as achievement of "clear" or "almost clear" skin. As such, duration of posttreatment maintenance of effect may have been greater if all participants had been allowed to continue treatment until achieving clear skin. This is supported by an analysis demonstrating that over $80 \%$ of participants who achieved clear skin did not require retreatment for over 1 month and almost 30\% did not require any retreatment for the duration of the study (Fig. 5) [88]. These results highlight the importance of treating to clear skin, which also reflects patient and clinician goals in a real-world setting.

\section{CONCLUSION}

Psoriasis is a chronic disease that frequently recurs after cessation of treatment, highlighting the need for therapies that provide rapid, robust, and lasting results while minimizing safety concerns and burden of use. HP $0.01 \%$ / TAZ $0.045 \%$ lotion is the only topical fixedcombination corticosteroid/tazarotene formulation approved for the treatment of psoriasis [89]. The complementary mechanisms of action of TAZ and HP, along with simultaneous delivery of moisturizers and hydrating agents, yields improvements in efficacy, prolonged duration of remission, and reductions in safety concerns with HP $0.01 \% / \mathrm{TAZ} \quad 0.045 \%$ lotion over monotherapy with either active ingredient, and support its use in the long-term management of psoriasis.

\section{ACKNOWLEDGEMENTS}

Funding. Sponsorship of this article and the journal's Rapid Service Fee were funded by Ortho Dermatologics (Bridgewater, NJ, USA). Ortho Dermatologics is a division of Bausch Health US, LLC.

Medical Writing/Editorial Assistance. Medical writing support was provided by Kevin Corcoran, PhD and Jacqueline Benjamin, PhD, of Prescott Medical Communications Group (Chicago, IL) with financial support from Ortho Dermatologics.

Authorship. All named authors meet the International Committee of Medical Journal Editors (ICMJE) criteria for authorship for this article, take responsibility for the integrity of the work as a whole, and have given their approval for this version to be published.

Author Contributions. All authors were involved in the interpretation of data and the critical review of all manuscript drafts. All authors approved the final manuscript draft for submission and are responsible for the integrity of this work.

Disclosures. Mark G. Lebwohl is an employee of Mount Sinai and receives research funds from: AbbVie, Amgen, AnaptysBio, 
Arcutis, Boehringer Ingelheim, Dermavant, Eli Lilly, Incyte, Janssen Research \& Development, LLC, LEO Pharma, Ortho Dermatologics, Pfizer, and UCB; is a consultant for Aditum Bio, Allergan, Almirall, Arcutis, Inc., Avotres Therapeutics, BirchBioMed Inc., BMD skincare, Boehringer-Ingelheim, Bristol-Myers Squibb, Cara Therapeutics, Castle Biosciences, Corrona, Dermavant Sciences, Evelo, Facilitate International Dermatologic Education, Foundation for Research and Education in Dermatology, Inozyme Pharma, Kyowa Kirin, LEO Pharma, Meiji Seika Pharma, Menlo, Mitsubishi, Neuroderm, Pfizer, Promius/Dr. Reddy's Laboratories, Serono, Theravance, and Verrica. Emil A. Tanghetti has served as speaker for Novartis, Ortho Dermatologics, Sun Pharma, Lilly, Galderma, AbbVie, and Dermira; served as a consultant/clinical studies for Hologic, Ortho Dermatologics, and Galderma; and is a stockholder for Accure. Linda Stein Gold has served as investigator/consultant or speaker for Ortho Dermatologics, LEO Pharma, Dermavant, Incyte, Novartis, AbbVie, Pfizer, Sun Pharma, UCB, Arcutis and Lilly. James Q. Del Rosso has served as a consultant, investigator, and/or speaker for Ortho Dermatologics, Amgen, Arcutis, Dermavant, EPI Heath, Galderma, Incyte, LEO Pharma, Lilly, MC2 Therapeutics, Pfizer, SunPharma, and UCB. Nelly K. Gilyadov and Abby Jacobson are employees of Ortho Dermatologics and may hold stock and/or stock options in its parent company.

Compliance with Ethics Guidelines. This article is based on previously conducted studies and does not contain any new studies with human participants or animals performed by any of the authors.

Open Access. This article is licensed under a Creative Commons Attribution-NonCommercial 4.0 International License, which permits any non-commercial use, sharing, adaptation, distribution and reproduction in any medium or format, as long as you give appropriate credit to the original author(s) and the source, provide a link to the Creative Commons licence, and indicate if changes were made. The images or other third party material in this article are included in the article's Creative Commons licence, unless indicated otherwise in a credit line to the material. If material is not included in the article's Creative Commons licence and your intended use is not permitted by statutory regulation or exceeds the permitted use, you will need to obtain permission directly from the copyright holder. To view a copy of this licence, visit http://creativecommons.org/licenses/by$\mathrm{nc} / 4.0 /$.

\section{REFERENCES}

1. Benhadou F, Mintoff D, Del Marmol V. Psoriasis: keratinocytes or immune cells-which is the trigger? Dermatology. 2019;235(2):91-100.

2. Nestle FO, Kaplan DH, Barker J. Psoriasis. N Engl J Med. 2009;361(5):496-509.

3. Wolf R, Orion E, Ruocco E, Ruocco V. Abnormal epidermal barrier in the pathogenesis of psoriasis. Clin Dermatol. 2012;30(3):323-8.

4. Menter A, Korman NJ, Elmets CA, et al. Guidelines of care for the management of psoriasis and psoriatic arthritis. Section 3. Guidelines of care for the management and treatment of psoriasis with topical therapies. J Am Acad Dermatol. 2009;60(4): 643-59.

5. Elmets CA, Korman NJ, Prater EF, et al. Joint AADNPF guidelines of care for the management and treatment of psoriasis with topical therapy and alternative medicine modalities for psoriasis severity measures. J Am Acad Dermatol. 2020;84:432-70.

6. Menter A, Strober BE, Kaplan DH, et al. Joint AADNPF guidelines of care for the management and treatment of psoriasis with biologics. J Am Acad Dermatol. 2019;80(4):1029-72.

7. Stein Gold LF, Kircik LH, Pariser DM. Understanding topical therapies for psoriasis. Cutis. 2019;103(4S):S8-12.

8. Tanghetti E, Lebwohl M, Stein GL. Tazarotene revisited: safety and efficacy in plaque psoriasis and its emerging role in treatment strategy. J Drugs Dermatol. 2018;17(12):1280-7.

9. Uva L, Miguel D, Pinheiro C, et al. Mechanisms of action of topical corticosteroids in psoriasis. Int J Endocrinol. 2012;2012:561018. 
10. Del Rosso JQ, Kim GK. The rationale behind topical vitamin $\mathrm{D}$ analogs in the treatment of psoriasis: where does topical calcitriol fit in? J Clin Aesthet Dermatol. 2010;3(8):46-53.

11. Bagel J, Thibodeaux QG, Han G. Halobetasol propionate for the management of psoriasis. Cutis. 2020;105(2):92-6.E4.

12. Weinstein GD. Tazarotene gel: efficacy and safety in plaque psoriasis. J Am Acad Dermatol. 1997;37(2 Pt 3):S33-8.

13. [Data on file]. Bridgewater (NJ): Ortho Dermatologics; 2021.

14. Tanghetti EA, Stein Gold L, Del Rosso JQ, Lin T, Angel A, Pillai R. Optimized formulation for topical application of a fixed combination halobetasol/tazarotene lotion using polymeric emulsion technology. J Dermatol Treat. 2019;3:1-8.

15. Chandraratna RA. Tazarotene: the first receptor-selective topical retinoid for the treatment of psoriasis. J Am Acad Dermatol. 1997;37(2 Pt 3):S12-7.

16. Duvic M, Nagpal S, Asano AT, Chandraratna RA. Molecular mechanisms of tazarotene action in psoriasis. J Am Acad Dermatol. 1997;37(2 Pt 3):S1824 .

17. Jeon SY, Ha SM, Ko DY, et al. Tazarotene-induced gene 3 may affect inflammatory angiogenesis in psoriasis by downregulating placental growth factor expression. Ann Dermatol. 2014;26(4):517-20.

18. Ohnishi S, Okabe $\mathrm{K}$, Obata $\mathrm{H}$, et al. Involvement of tazarotene-induced gene 1 in proliferation and differentiation of human adipose tissue-derived mesenchymal stem cells. Cell Prolif. 2009;42(3):309-16.

19. Scharadin TM, Eckert RL. Tig3: an important regulator of keratinocyte proliferation and survival. J Invest Dermatol. 2014;134(7):1811-6.

20. Duvic M, Helekar B, Schulz C, et al. Expression of a retinoid-inducible tumor suppressor, tazaroteneinducible gene-3, is decreased in psoriasis and skin cancer. Clin Cancer Res. 2000;6(8):3249-59.

21. Duvic M, Asano AT, Hager C, Mays S. The pathogenesis of psoriasis and the mechanism of action of tazarotene. J Am Acad Dermatol. 1998;39(4 Pt 2): S129-33.

22. Zhou XF, Shen XQ, Shemshedini L. Ligand-activated retinoic acid receptor inhibits $\mathrm{AP}-1$ transactivation by disrupting c-Jun/c-Fos dimerization. Mol Endocrinol. 1999;13(2):276-85.

23. Tsai EY, Jain J, Pesavento PA, Rao A, Goldfeld AE. Tumor necrosis factor alpha gene regulation in activated T cells involves ATF-2/Jun and NFATp. Mol Cell Biol. 1996;16(2):459-67.

24. Chan SC, Brown MA, Willcox TM, et al. Abnormal IL-4 gene expression by atopic dermatitis T lymphocytes is reflected in altered nuclear protein interactions with IL-4 transcriptional regulatory element. J Invest Dermatol. 1996;106(5):1131-6.

25. Samten B, Townsend JC, Weis SE, et al. CREB, ATF, and AP-1 transcription factors regulate IFN-gamma secretion by human $\mathrm{T}$ cells in response to mycobacterial antigen. J Immunol. 2008;181(3): 2056-64.

26. Baliwag J, Barnes DH, Johnston A. Cytokines in psoriasis. Cytokine. 2015;73(2):342-50.

27. Swindell WR, Johnston A, Voorhees JJ, Elder JT, Gudjonsson JE. Dissecting the psoriasis transcriptome: inflammatory- and cytokine-driven gene expression in lesions from 163 patients. BMC Genom. 2013;14:527.

28. Nagpal S, Athanikar J, Chandraratna RA. Separation of transactivation and Ap1 antagonism functions of retinoic acid receptor alpha. J Biol Chem. 1995;270(2):923-7.

29. Weng JR, Huang TH, Lin ZC, Alalaiwe A, Fang JY. Cutaneous delivery of [1-(4-chloro-3-nitrobenzenesulfonyl)-1H-indol-3-yl]-methanol, an indole-3carbinol derivative, mitigates psoriasiform lesion by blocking MAPK/NF-kB/AP-1 activation. Biomed Pharmacother. 2019;119:109398.

30. King LE Jr, Gates RE, Stoscheck CM, Nanney LB. Epidermal growth factor/transforming growth factor alpha receptors and psoriasis. J Invest Dermatol. 1990;95(5 Suppl):10S-S12.

31. Elder JT, Fisher GJ, Lindquist PB, et al. Overexpression of transforming growth factor alpha in psoriatic epidermis. Science. 1989;243(4892):811-4.

32. Esgleyes-Ribot T, Chandraratna RA, Lew-Kaya DA, Sefton J, Duvic M. Response of psoriasis to a new topical retinoid, AGN 190168. J Am Acad Dermatol. 1994;30(4):581-90.

33. Lee AY. Molecular mechanism of epidermal barrier dysfunction as primary abnormalities. Int J Mol Sci. 2020;21(4):1194.

34. Griffiths CE, Voorhees JJ, Nickoloff BJ. Characterization of intercellular adhesion molecule- 1 and HLA-DR expression in normal and inflamed skin: modulation by recombinant gamma interferon and tumor necrosis factor. J Am Acad Dermatol. 1989;20(4):617-29. 
35. Apisarnthanarax N, Talpur R, Ward S, Ni X, Kim HW, Duvic M. Tazarotene $0.1 \%$ gel for refractory mycosis fungoides lesions: an open-label pilot study. J Am Acad Dermatol. 2004;50(4):600-7.

36. Nukui T, Ehama R, Sakaguchi M, et al. S100A8/A9, a key mediator for positive feedback growth stimulation of normal human keratinocytes. J Cell Biochem. 2008;104(2):453-64.

37. Lee DG, Woo JW, Kwok SK, Cho ML, Park SH. MRP8 promotes Th17 differentiation via upregulation of IL-6 production by fibroblast-like synoviocytes in rheumatoid arthritis. Exp Mol Med. 2013;45:e20.

38. Nagpal S, Patel S, Jacobe $H$, et al. Tazarotene-induced gene 2 (TIG2), a novel retinoid-responsive gene in skin. J Invest Dermatol. 1997;109(1):91-5.

39. Shin WJ, Zabel BA, Pachynski RK. Mechanisms and functions of chemerin in cancer: potential roles in therapeutic intervention. Front Immunol. 2018;9: 2772.

40. Li J, Lu Y, Li N, et al. Chemerin: a potential regulator of inflammation and metabolism for chronic obstructive pulmonary disease and pulmonary rehabilitation. Biomed Res Int. 2020;2020:4574509.

41. Chiricozzi A, Romanelli P, Volpe E, Borsellino G, Romanelli M. Scanning the immunopathogenesis of psoriasis. Int J Mol Sci. 2018;19(1):179.

42. Albanesi C, Scarponi C, Pallotta S, et al. Chemerin expression marks early psoriatic skin lesions and correlates with plasmacytoid dendritic cell recruitment. J Exp Med. 2009;206(1):249-58.

43. Barnes PJ. Anti-inflammatory actions of glucocorticoids: molecular mechanisms. Clin Sci (Lond). 1998;94(6):557-72.

44. Hengge UR, Ruzicka T, Schwartz RA, Cork MJ. Adverse effects of topical glucocorticosteroids. J Am Acad Dermatol. 2006;54(1):1-15 (quiz 6-8).

45. Brazzini B, Pimpinelli N. New and established topical corticosteroids in dermatology: clinical pharmacology and therapeutic use. Am J Clin Dermatol. 2002;3(1):47-58.

46. Greaves MW. Anti-inflammatory action of corticosteroids. Postgrad Med J. 1976;52(612):631-3.

47. Ahluwalia A. Topical glucocorticoids and the skin-mechanisms of action: an update. Mediat Inflamm. 1998;7(3):183-93.

48. Gabros S, Nessel TA, Zito PM. Topical corticosteroids. Statpearls [internet]. Treasure Island (FL). https://www.ncbi.nlm.nih.gov/books/NBK532940. 2020.
49. Niculet E, Bobeica C, Tatu AL. Glucocorticoid-induced skin atrophy: the old and the new. Clin Cosmet Investig Dermatol. 2020;13:1041-50.

50. Lebwohl MG, Breneman DL, Goffe BS, et al. Tazarotene $0.1 \%$ gel plus corticosteroid cream in the treatment of plaque psoriasis. J Am Acad Dermatol. 1998;39(4 Pt 1):590-6.

51. Koo JY, Martin D. Investigator-masked comparison of tazarotene gel q.d. plus mometasone furoate cream q.d. vs mometasone furoate cream b.i.d. In the treatment of plaque psoriasis. Int J Dermatol. $2001 ; 40(3): 210-2$.

52. Lebwohl M. Strategies to optimize efficacy, duration of remission, and safety in the treatment of plaque psoriasis by using tazarotene in combination with a corticosteroid. J Am Acad Dermatol. 2000;43(2 Pt 3):S43-6.

53. Yin S, Luo J, Qian A, et al. Retinoids activate the irritant receptor TRPV1 and produce sensory hypersensitivity. J Clin Invest. 2013;123(9): 3941-51.

54. Ahn S, Park J, An I, Jung SJ, Hwang J. Transient receptor potential cation channel V1 (TRPV1) is degraded by starvation- and glucocorticoid-mediated autophagy. Mol Cells. 2014;37(3):257-63.

55. McMichael AJ, Griffiths CE, Talwar HS, et al. Concurrent application of tretinoin (retinoic acid) partially protects against corticosteroid-induced epidermal atrophy. Br J Dermatol. 1996;135(1): $60-4$.

56. Ogden S, Samuel M, Griffiths CE. A review of tazarotene in the treatment of photodamaged skin. Clin Interv Aging. 2008;3(1):71-6.

57. Kaidbey K, Kopper SC, Sefton J, Gibson JR. A pilot study to determine the effect of tazarotene gel $0.1 \%$ on steroid-induced epidermal atrophy. Int J Dermatol. 2001;40(7):468-71.

58. Cornell RC, Stoughton RB. Correlation of the vasoconstriction assay and clinical activity in psoriasis. Arch Dermatol. 1985;121(1):63-7.

59. Tan X, Feldman SR, Chang J, Balkrishnan R. Topical drug delivery systems in dermatology: a review of patient adherence issues. Expert Opin Drug Deliv. 2012;9(10):1263-71.

60. Yu B, Ma P, Yuan L, Chen D, Yang J. Evaluation of change in the skin concentration of tazarotene and betamethasone dipropionate based on drug-drug interaction for transdermal drug delivery in miniature pig. Xenobiotica. 2015;45(5):380-4. 
61. Bikowski JB. Barrier repair as an element of psoriasis management. Pract Dermatol. 2010(May):32-3.

62. Thiboutot D, Del Rosso JQ. Acne vulgaris and the epidermal barrier. J Clin Aesthet Dermatol. 2013;6(2):18-24.

63. Del Rosso JQ, Cash K. Topical corticosteroid application and the structural and functional integrity of the epidermal barrier. J Clin Aesthet Dermatol. 2013;6(11):20-7.

64. Draelos ZD, Ertel KD, Berge CA. Facilitating facial retinization through barrier improvement. Cutis. 2006;78(4):275-81.

65. Motta S, Monti M, Sesana S, Mellesi L, Ghidoni R, Caputo R. Abnormality of water barrier function in psoriasis. Role of ceramide fractions. Arch Dermatol. $1994 ; 130(4): 452-6$.

66. Eastman WJ, Malahias S, Delconte J, DiBenedetti D. Assessing attributes of topical vehicles for the treatment of acne, atopic dermatitis, and plaque psoriasis. Cutis. 2014;94(1):46-53.

67. Svendsen MT, Feldman SR, Tiedemann SN, Sorensen ASS, Rivas CMR, Andersen KE. Psoriasis patient preferences for topical drugs: a systematic review. J Dermatolog Treat. 2019;1-6.

68. Housman TS, Mellen BG, Rapp SR, Fleischer AB Jr, Feldman SR. Patients with psoriasis prefer solution and foam vehicles: a quantitative assessment of vehicle preference. Cutis. 2002;70(6):327-32.

69. Draelos ZD, Tanghetti EA. A comparative clinical demonstration of the spreadability of tazarotene lotion $0.045 \%$ versus trifarotene cream $0.005 \%$. Fall Clinical Oct 29-Nov 12020.

70. Sugarman JL, Gold LS, Lebwohl MG, Pariser DM, Alexander BJ, Pillai R. A phase 2, multicenter, double-blind, randomized, vehicle controlled clinical study to assess the safety and efficacy of a halobetasol/tazarotene fixed combination in the treatment of plaque psoriasis. J Drugs Dermatol. 2017;16(3):197-204.

71. Sugarman JL, Weiss J, Tanghetti EA, et al. Safety and efficacy of a fixed combination halobetasol and tazarotene lotion in the treatment of moderate-tosevere plaque psoriasis: a pooled analysis of two phase 3 studies. J Drugs Dermatol. 2018;17(8): 855-61.

72. Stein Gold L, Lebwohl MG, Sugarman JL, et al. Safety and efficacy of a fixed combination of halobetasol and tazarotene in the treatment of moderate-to-severe plaque psoriasis: results of 2 phase 3 randomized controlled trials. J Am Acad Dermatol. 2018;79(2):287-93.
73. Lebwohl MG, Stein Gold L, Papp K, et al. Long-term safety and efficacy of a fixed-combination halobetasol propionate $00.1 \%$ /tazarotene $0.045 \%$ lotion in moderate-to-severe plaque psoriasis: phase 3 openlabel study. J Eur Acad Dermatol Venereol. 2021;35(5):1152-60.

74. Stein Gold L, Elewski B, Draelos Z, Jacobson A, Lin T. Halobetasol $00.1 \% /$ tazarotene $0.045 \%$ lotion for moderate-to-severe psoriasis: pooled phase 3 analysis of males and females. J Drugs Dermatol. 2020;19(5):504-14.

75. Alexis AF, Yamauchi PS, Desai SR, Khaselev N, Lin T. Efficacy, safety, and tolerablity of halobetasol propionate $0.01 \%$-tazarotene $0.045 \%$ lotion for moderate to severe plaque psoriasis in the hispanic population: post hoc analysis. Cutis. 2020;105(3): 150-5.

76. Tyring S, Kircik LH, Yamauchi P, Jacobson A, Lin T. Halobetasol propionate $0.01 \%$ /tazarotene $0.045 \%$ lotion for moderate-to-severe psoriasis: pooled phase 3 analysis of lower extremities. J Drugs Dermatol. 2020;19(4):389-96.

77. Leonardi C, Stein Gold L, Lain E, Neimann A, Jacobson A. Halobetasol 0.01\%/tazarotene 0.045\% (hp/taz) lotion for the treatment of plaque psoriasis in patients with $3-5 \%$ body surface area. Poster presented at Fall Clinical Dermatology Conference. October 29-November 1, 2020.

78. Lebwohl MG, Sugarman JL, Gold LS, et al. Longterm safety results from a phase 3 open-label study of a fixed combination halobetasol propionate 0 . 01\% and tazarotene $0.045 \%$ lotion in moderate-tosevere plaque psoriasis. J Am Acad Dermatol. 2019;80(1):282-5.

79. Castela E, Archier E, Devaux S, et al. Topical corticosteroids in plaque psoriasis: a systematic review of risk of adrenal axis suppression and skin atrophy. J Eur Acad Dermatol Venereol. 2012;26(Suppl 3): 47-51.

80. Griffiths CE, Voorhees JJ. Human in vivo pharmacology of topical retinoids. Arch Dermatol Res. 1994;287(1):53-60.

81. Rossetti D, Kielmanowicz MG, Vigodman S, et al. A novel anti-ageing mechanism for retinol: induction of dermal elastin synthesis and elastin fibre formation. Int J Cosmet Sci. 2011;33(1):62-9.

82. Dando TM, Wellington K. Topical tazarotene: a review of its use in the treatment of plaque psoriasis. Am J Clin Dermatol. 2005;6(4):255-72.

83. Duobrii [package insert]. Bridgewater, NJ: Ortho Dermatologics; 2020. 
84. Tan J, Gollnick HP, Loesche C, Ma YM, Gold LS. Synergistic efficacy of adapalene 0.1\%-benzoyl peroxide $2.5 \%$ in the treatment of 3855 acne vulgaris patients. J Dermatol Treat. 2011;22(4): 197-205.

85. Kircik LH, Papp KA, Stein Gold L, Harris S, Pharm TL, Pillai R. Assessing the synergistic effect of a fixed combination halobetasol propionate $0.01 \%$ and tazarotene $0.045 \%$ lotion in moderate-to-severe plaque psoriasis. J Drugs Dermatol. 2019;18(3): 279-84.

86. Weinstein GD, Koo JY, Krueger GG, et al. Tazarotene cream in the treatment of psoriasis: two multicenter, double-blind, randomized, vehiclecontrolled studies of the safety and efficacy of tazarotene creams $0.05 \%$ and $0.1 \%$ applied once daily for 12 weeks. J Am Acad Dermatol. 2003;48(5): 760-7.

87. Stein Gold L, Lain E, Green LJ, Lin T, Israel R. Halobetasol $0.01 \% /$ tazarotene $0.045 \%$ lotion in the treatment of moderate-to-severe plaque psoriasis: maintenance of therapeutic effect after cessation of therapy. J Drugs Dermatol. 2019;18(8):815-20.

88. Stein Gold L, Lebwohl M, Bhatia N, DiRuggiero D, Jacobson A, Pillai R. Long-term management of moderate-to-severe plaque psoriasis: maintenance of treatment success following cessation of halobetasol propionate $0.01 \% /$ tazarotene $0.045 \%$ lotion. Skin. 2020;4(6):s79.

89. Expanded table: Some drugs for psoriasis. Med Lett Drugs Ther. 2019;61(1574):e96-e103. 